\title{
Some Convergence and Stability Results for the Kirk Multistep and Kirk-SP Fixed Point Iterative Algorithms
}

\author{
Faik Gürsoy, ${ }^{1}$ Vatan Karakaya, ${ }^{2}$ and B. E. Rhoades ${ }^{3}$ \\ ${ }^{1}$ Department of Mathematics, Yildiz Technical University, Davutpasa Campus, Esenler, 34220 Istanbul, Turkey \\ ${ }^{2}$ Department of Mathematical Engineering, Yildiz Technical University, Davutpasa Campus, Esenler, 34210 Istanbul, Turkey \\ ${ }^{3}$ Department of Mathematics, Indiana University, Bloomington, IN 47405-7106, USA
}

Correspondence should be addressed to Faik Gürsoy; faikgursoy02@hotmail.com

Received 1 July 2013; Accepted 10 November 2013; Published 3 February 2014

Academic Editor: Micah Osilike

Copyright (C) 2014 Faik Gürsoy et al. This is an open access article distributed under the Creative Commons Attribution License, which permits unrestricted use, distribution, and reproduction in any medium, provided the original work is properly cited.

The purpose of this paper is to introduce a new Kirk type iterative algorithm called Kirk multistep iteration and to study its convergence. We also prove some theorems related to the stability results for the Kirk multistep and Kirk-SP iterative processes by employing certain contractive-like operators. Our results generalize and unify some other results in the literature.

\section{Introduction and Preliminaries}

This paper is organized as follows. Section 1 outlines some known contractive mappings and iterative schemes and collects some preliminaries that will be used in the proofs of our main results. We then propose a new Kirk type iterative process called Kirk multistep iteration. Section 2 presents a result dealing with the convergence of this new iterative procedure, which unifies and extends some other iterative schemes in the existing literature. Also we prove some theorems related to the stability of the Kirk multistep and Kirk-SP iterative processes by employing certain contractivelike operators.

Fixed point iterations are commonly used to solve nonlinear equations arising in physical systems. Such equations can be transformed into a fixed point equation $T x=x$ which is solved by some iterative processes of form $x_{n+1}=f\left(T, x_{n}\right)$, $n=0,1,2, \ldots$, that converges to a fixed point of $T$. This is a reason, among a number of reasons, why there is presently a great deal of interest in the introduction and development of various iterative algorithms. Consequently iteration schemes abound in the literature of fixed point theory, for which fixed points of operators have been approximated over the years by various authors, for example, [1-10].

As a background to our exposition, we describe some iteration schemes and contractive type mappings.
Throughout this paper $\mathbb{N}$ denotes the nonnegative integers, including zero. Let $\left\{\alpha_{n}\right\}_{n=0}^{\infty},\left\{\beta_{n}\right\}_{n=0}^{\infty},\left\{\gamma_{n}\right\}_{n=0}^{\infty}$, and $\left\{\beta_{n}^{i}\right\}_{n=0}^{\infty}$, $i=\overline{1, k-2}, k \geq 2$, be real sequences in $[0,1)$ satisfying certain conditions.

Rhoades and Şoltuz [1] introduced a multistep iterative algorithm by

$$
\begin{gathered}
x_{0} \in E, \\
x_{n+1}=\left(1-\alpha_{n}\right) x_{n}+\alpha_{n} T y_{n}^{1}, \\
y_{n}^{i}=\left(1-\beta_{n}^{i}\right) x_{n}+\beta_{n}^{i} T y_{n}^{i+1}, \\
y_{n}^{k-1}=\left(1-\beta_{n}^{k-1}\right) x_{n}+\beta_{n}^{k-1} T x_{n}, \quad n \in \mathbb{N} .
\end{gathered}
$$

The following multistep iteration was employed in $[2,11]$ :

$$
\begin{gathered}
x_{0} \in E, \\
x_{n+1}=\left(1-\alpha_{n}\right) y_{n}^{1}+\alpha_{n} T y_{n}^{1}, \\
y_{n}^{i}=\left(1-\beta_{n}^{i}\right) y_{n}^{i+1}+\beta_{n}^{i} T y_{n}^{i+1}, \\
y_{n}^{k-1}=\left(1-\beta_{n}^{k-1}\right) x_{n}+\beta_{n}^{k-1} T x_{n}, \quad n \in \mathbb{N} .
\end{gathered}
$$

By taking $k=3$ and $k=2$ in (1) we obtain the well-known Noor [4] and Ishikawa [6] iterative schemes, respectively. SP 
iteration [8] and a new two-step iteration [7] processes are obtained by taking $k=3$ and $k=2$ in (2), respectively. Both in (1) and in (2), if we take $k=2$ with $\beta_{n}^{1}=0$ and $k=2$ with $\beta_{n}^{1} \equiv 0, \alpha_{n} \equiv \lambda$ (const.), then we get the iterative procedures introduced in $[10,12]$, which are commonly known as the Mann and Krasnoselskii iterations, respectively. The Krasnoselskii iteration reduces to the Picard iteration [13] for $\lambda=1$.

The Kirk-SP iterative scheme [14] is defined by

$$
\begin{gathered}
x_{n+1}=\sum_{i_{1}=0}^{s_{1}} \alpha_{n, i_{1}} T^{i_{1}} y_{n}^{1}, \quad \sum_{i_{1}=0}^{s_{1}} \alpha_{n, i_{1}}=1, \\
y_{n}^{1}=\sum_{i_{2}=0}^{s_{2}} \beta_{n, i_{2}}^{1} T^{i_{2}} y_{n}^{2}, \quad \sum_{i_{2}=0}^{s_{2}} \beta_{n, i_{2}}^{1}=1, \\
y_{n}^{2}=\sum_{i_{3}=0}^{s_{3}} \beta_{n, i_{3}}^{2} T^{i_{3}} x_{n}, \quad \sum_{i_{3}=0}^{s_{3}} \beta_{n, i_{3}}^{2}=1, \quad \forall n \in \mathbb{N},
\end{gathered}
$$

where $s_{1}, s_{2}$, and $s_{3}$ are fixed integers with $s_{1} \geq s_{2} \geq s_{3}$ and $\alpha_{n, i_{1}}, \beta_{n, i_{2}}^{1}, \beta_{n, i_{3}}^{2}$ are sequences in $[0,1]$ satisfying $\alpha_{n, i_{1}} \geq 0$, $\alpha_{n, 0} \neq 0, \beta_{n, i_{2}}^{1} \geq 0, \beta_{n, 0}^{1} \neq 0, \beta_{n, i_{3}}^{2} \geq 0, \beta_{n, 0}^{2} \neq 0$.

Let $X$ be an arbitrary Banach space and $T: X \rightarrow X$ a mapping.

We will introduce and employ the following iterative scheme, which is called a Kirk multistep iteration:

$$
\begin{gathered}
x_{0} \in X, \\
x_{n+1}=\alpha_{n, 0} x_{n}+\sum_{i_{1}=1}^{s_{1}} \alpha_{n, i_{1}} T^{i_{1}} y_{n}^{1}, \\
y_{n}^{p}=\beta_{n, 0}^{p} x_{n}+\sum_{i_{p+1}=1}^{s_{p+1}} \beta_{n, i_{p+1}}^{p} T^{i_{p+1}} y_{n}^{p+1}, \quad p=\overline{1, k-2}, \\
y_{n}^{k-1}=\sum_{i_{k}=0}^{s_{k}} \beta_{n, i_{k}}^{k-1} T^{i_{k}} x_{n}, \quad k \geq 2, \forall n \in \mathbb{N},
\end{gathered}
$$

where $\sum_{i_{1}=0}^{s_{1}} \alpha_{n, i_{1}}=1, \sum_{i_{p+1}=0}^{s_{p+1}} \beta_{n, i_{p+1}}^{p}=1$ for $p=\overline{1, k-1}$; $\alpha_{n, i_{1}}, \beta_{n, i_{p+1}}^{p}$ are sequences in $[0,1]$ satisfying $\alpha_{n, i_{1}} \geq 0, \alpha_{n, 0} \neq 0$, $\beta_{n, i_{p+1}}^{p} \geq 0, \beta_{n, 0}^{p} \neq 0$ for $p=\overline{1, k-1}$ and $s_{1}, s_{p+1}$ for $p=\overline{1, k-1}$ are fixed integers with $s_{1} \geq s_{2} \geq \cdots \geq s_{k}$.

By taking $k=3, k=2$, and $k=2$ with $s_{2}=0$ in (4) we obtain the Kirk-Noor [15], the Kirk-Ishikawa [16] and the Kirk-Mann [16], iterative schemes, respectively. Also, (4) gives the usual Kirk iterative process [17] for $k=2$, with $s_{2}=0$ and $\alpha_{n, i_{1}}=\alpha_{i_{1}}$. If we put $s_{1}=1$ and $s_{p+1}=1, p=\overline{1, k-1}$ in (4), then we have the usual multistep iteration (1) with $\sum_{i_{1}=0}^{1} \alpha_{n, i_{1}}=1, \alpha_{n, 1}=\alpha_{n}, \sum_{i_{p+1}=0}^{1} \beta_{n, i_{p+1}}^{p}=1, \beta_{n, 1}^{p}=\beta_{n}^{p}, p=$ $\overline{1, k-1}$. The Noor iteration [4], the Ishikawa iteration [6], the Mann iteration [10], the Krasnoselskii iteration [12], and the Picard iteration [13] schemes are special cases of the multistep iterative scheme (1), as explained above. So, we conclude that these are special cases of the Kirk multistep iterative scheme (4).

A particular fixed point iteration generates a theoretical sequence $\left\{x_{n}\right\}_{n=0}^{\infty}$. In applications, various errors (e.g., roundoff or discretization of the function $T$ etc.) occur during computation of the sequence $\left\{x_{n}\right\}_{n=0}^{\infty}$. Because of these errors we cannot obtain the theoretical sequence $\left\{x_{n}\right\}_{n=0}^{\infty}$, but an approximate sequence $\left\{y_{n}\right\}_{n=0}^{\infty}$ instead. We will say that the iterative process is $T$-stable or stable with respect to $T$ if and only if $\left\{x_{n}\right\}_{n=0}^{\infty}$ converges to a fixed point $q$ of $T$, then $\left\{y_{n}\right\}_{n=0}^{\infty}$ converges to $q=T q$.

The initiator of this kind study is Urabe [18] while a formal definition for the stability of general iterative schemes is given by Harder and Hicks $[19,20]$ as follows.

Definition 1. Let $(X, d)$ be a complete metric space and $T$ a self-map of $X$. Suppose that $F_{T}=\{q \in X: q=T q\}$ is the set of fixed points of $T$. Let $\left\{x_{n}\right\}_{n=0}^{\infty} \subset X$ be a sequence generated by an iterative process defined by

$$
x_{n+1}=f\left(T, x_{n}\right), \quad n=0,1, \ldots,
$$

where $x_{0} \in X$ is the initial approximation and $f$ is some function. Let $\left\{y_{n}\right\}_{n=0}^{\infty} \subset X$ be an arbitrary sequence and set $\varepsilon_{n}=d\left(y_{n+1}, f\left(T, y_{n}\right)\right), n=0,1, \ldots$ Then, the iterative process (5) is said to be $T$-stable or stable with respect to $T$ if and only if $\lim _{n \rightarrow \infty} \varepsilon_{n}=0 \Rightarrow \lim _{n \rightarrow \infty} y_{n}=q$.

In the last three decades, a large literature has developed dealing with the stability of various well-known iterative schemes for different classes of operators. Several authors who have made contributions to the study of stability of fixed point iterative procedures are Ostrowski [21], Harder [22], Harder and Hicks [19, 20], Rhoades [23, 24], Berinde $[25,26]$, Osilike [27, 28], Osilike and Udomene [29], Olatinwo $[16,30,31]$, Chugh and Kumar [15], and several references contained therein.

A pioneering result on the stability of iterative procedures established in metric space for the Picard iteration is due to Ostrowski [21], which states that: Let $(X, d)$ be a complete metric space and $T: X \rightarrow X$ a Banach contraction mapping, that is,

$$
d(T x, T y) \leq \lambda d(x, y) \quad \forall x, y \in X,
$$

where $\lambda \in[0,1)$. Let $q \in X$ be the fixed point of $T, x_{0} \in$ $X$, and $x_{n+1}=T x_{n}, n=0,1,2, \ldots$. Suppose that $\left\{y_{n}\right\}_{n=0}^{\infty}$ is a sequence in $X$ and $\varepsilon_{n}=d\left(y_{n+1}, T y_{n}\right)$. Then

$$
d\left(q, y_{n+1}\right) \leq d\left(q, x_{n+1}\right)+\lambda^{n+1} d\left(x_{0}, y_{0}\right)+\sum_{i=0}^{n} \lambda^{n-r} \varepsilon_{i} .
$$

Moreover, $\lim _{n \rightarrow \infty} y_{n}=q \Leftrightarrow \lim _{n \rightarrow \infty} \varepsilon_{n}=0$.

Using Definition 1, Harder and Hicks [19, 20] proved some stability theorems for well-known Picard, Mann, and Kirk's iterations by employing several classes of contractive type operators. Rhoades $[23,24]$ extended the results of Harder and Hicks [20] by utilizing the following two different classes of contractive operators of Ciric's type, 
respectively: there exists a $\lambda \in[0,1)$ such that for each pair $x, y \in X$

$$
\begin{gathered}
d(T x, T y) \leq \lambda \max \{d(x, y), d(x, T y), d(y, T x)\}, \\
d(T x, T y) \\
\leq \lambda \max \left\{d(x, y), \frac{\{d(x, T x)+d(y, T y)\}}{2},\right. \\
d(x, T y), d(y, T x)\} .
\end{gathered}
$$

Later Osilike [27] further generalized and extended some of the results in [23] by using a large class of contractive type operators $T$ satisfying the following condition, which is more general than those of Rhoades $[23,24]$ and Harder and Hicks [20]:

$$
d(T x, T y) \leq L d(x, T x)+\lambda d(x, y)
$$

for some $\lambda \in[0,1), L \geq 0$, and for all $x, y \in X$.

By employing the contractive condition (9), Osilike and Udomene proved some stability results for the Picard, Ishikawa, and Kirk's iteration in [29] where a new and shorter method than those mentioned above was used. Using the same method of proof as in [29], Berinde [26] again established the stability results in Harder and Hicks [20].

In [32], Imoru and Olatinwo extended some of the stability results of $[20,23,24,26,27,29]$ by employing a much more general class of operators $T$ satisfying the following contractive condition:

$$
d(T x, T y) \leq \varphi(d(x, T x))+\lambda d(x, y), \quad \forall x, y \in X,
$$

where $\lambda \in[0,1)$ and $\varphi: \mathbb{R}^{+} \rightarrow \mathbb{R}^{+}$is a monotone increasing function with $\varphi(0)=0$.

Remark 2 (see $[2,11])$. A map satisfying (10) need not have a fixed point. However, using (10), it is obvious that if $T$ has a fixed point, then it is unique.

Continuing the abovementioned trend, Olatinwo [16] studied the stability of the Kirk-Mann and Kirk-Ishikawa iterative processes by utilizing contractive condition (10). The results of [16] are generalizations of some of the results of [20, 23, 24, 26, 27, 29, 33-35].

Recently Chugh and Kumar [15] improved and extended the results of [16] and some of the references cited therein by introducing the Kirk-Noor iterative algorithm.

We end this section with some lemmas which will be useful in proving our main results.

Lemma 3 (see [36]). If $\sigma$ is a real number such that $\sigma \in[0,1)$, and $\left\{\varepsilon_{n}\right\}_{n=0}^{\infty}$ is a sequence of nonnegative numbers such that $\lim _{n \rightarrow \infty} \varepsilon_{n}=0$, then, for any sequence of positive numbers $\left\{u_{n}\right\}_{n=0}^{\infty}$ satisfying

$$
u_{n+1} \leq \sigma u_{n}+\varepsilon_{n}, \quad \forall n \in \mathbb{N},
$$

one has $\lim _{n \rightarrow \infty} u_{n}=0$.

Lemma 4 (see $[16])$. Let $(X,\|\cdot\|)$ be a normed linear space and $T$ a self-map of $X$ satisfying (10). Let $\varphi: \mathbb{R}^{+} \rightarrow \mathbb{R}^{+}$be a subadditive, monotone increasing function such that $\varphi(0)=0$, $\varphi(L u) \leq L \varphi(u), L \geq 0, u \in \mathbb{R}^{+}$. Then, for all $i \in \mathbb{N}, L \geq 0$ and for all $x, y \in X$

$$
\left\|T^{i} x-T^{i} y\right\| \leq \sum_{j=1}^{i}\left(\begin{array}{l}
i \\
j
\end{array}\right) a^{i-j} \varphi^{j}(\|x-T x\|)+a^{i}\|x-y\| .
$$

Remark 5. Note that $a \in[0,1)$ in the inequality (12).

\section{Main Results}

For simplicity we assume in the following three theorems that $X$ is a normed linear space, $T$ is a self-map of $X$ satisfying the contractive condition (10) with $F_{T} \neq \emptyset$, and $\varphi: \mathbb{R}^{+} \rightarrow \mathbb{R}^{+}$is a subadditive monotone increasing function such that $\varphi(0)=0$ and $\varphi(L u) \leq L \varphi(u), L \geq 0, u \in \mathbb{R}^{+}$.

Theorem 6. Let $\left\{x_{n}\right\}_{n \in \mathbb{N}}$ be a sequence generated by the Kirk multistep iterative scheme (4). Suppose that $T$ has a fixed point $q$. Then the iterative sequence $\left\{x_{n}\right\}_{n \in \mathbb{N}}$ converges strongly to $q$.

Proof. The uniqueness of $q$ follows from (12). We will now prove that $x_{n} \rightarrow q$.

Using (4) and Lemma 4, we get

$$
\begin{aligned}
& \left\|x_{n+1}-q\right\| \\
& =\left\|\alpha_{n, 0} x_{n}-\alpha_{n, 0} q+\sum_{i_{1}=1}^{s_{1}} \alpha_{n, i_{1}} T^{i_{1}} y_{n}^{1}-\sum_{i_{1}=1}^{s_{1}} \alpha_{n, i_{1}} q\right\| \\
& \leq \alpha_{n, 0}\left\|x_{n}-q\right\|+\sum_{i_{1}=1}^{s_{1}} \alpha_{n, i_{1}}\left\|T^{i_{1}} y_{n}^{1}-T^{i_{1}} q\right\| \\
& \leq \alpha_{n, 0}\left\|x_{n}-q\right\| \\
& +\sum_{i_{1}=1}^{s_{1}} \alpha_{n, i_{1}}\left\{\sum_{j=1}^{i_{1}}\left(\begin{array}{c}
i_{1} \\
j
\end{array}\right) a^{i_{1}-j} \varphi^{j}(\|q-T q\|)\right. \\
& \left.+a^{i_{1}}\left\|y_{n}^{1}-q\right\|\right\} \\
& =\alpha_{n, 0}\left\|x_{n}-q\right\|+\left(\sum_{i_{1}=1}^{s_{1}} \alpha_{n, i_{1}} a^{i_{1}}\right)\left\|y_{n}^{1}-q\right\|,
\end{aligned}
$$


4

Abstract and Applied Analysis

$$
\begin{aligned}
& \left\|y_{n}^{1}-q\right\| \\
& =\left\|\beta_{n, 0}^{1} x_{n}+\sum_{i_{2}=1}^{s_{2}} \beta_{n, i_{2}}^{1} T^{i_{2}} y_{n}^{2}-\sum_{i_{2}=0}^{s_{2}} \beta_{n, i_{2}}^{1} q\right\| \\
& \quad \leq \beta_{n, 0}^{1}\left\|x_{n}-q\right\|+\sum_{i_{2}=1}^{s_{2}} \beta_{n, i_{2}}^{1}\left\|T^{i_{2}} y_{n}^{2}-T^{i_{2}} q\right\|
\end{aligned}
$$$$
\leq \beta_{n, 0}^{1}\left\|x_{n}-q\right\|
$$

By combining (13), (14), (15), and (16) we obtain

$$
\begin{aligned}
\| x_{n+1} & -q \| \\
\leq & \left\{\alpha_{n, 0}+\left(\sum_{i_{1}=1}^{s_{1}} \alpha_{n, i_{1}} a^{i_{1}}\right) \beta_{n, 0}^{1}+\left(\sum_{i_{1}=1}^{s_{1}} \alpha_{n, i_{1}} a^{i_{1}}\right)\right. \\
& \times\left(\sum_{i_{2}=1}^{s_{2}} \beta_{n, i_{2}}^{1} a^{i_{2}}\right) \beta_{n, 0}^{2}+\left(\sum_{i_{1}=1}^{s_{1}} \alpha_{n, i_{1}} a^{i_{1}}\right) \\
& \left.\times\left(\sum_{i_{2}=1}^{s_{2}} \beta_{n, i_{2}}^{1} a^{i_{2}}\right)\left(\sum_{i_{3}=1}^{s_{3}} \beta_{n, i_{3}}^{2} a^{i_{3}}\right) \beta_{n, 0}^{3}\right\}\left\|x_{n}-q\right\| \\
& +\left(\sum_{i_{1}=1}^{s_{1}} \alpha_{n, i_{1}} a^{i_{1}}\right)\left(\sum_{i_{2}=1}^{s_{2}} \beta_{n, i_{2}}^{1} a^{i_{2}}\right) \\
& \times\left(\sum_{i_{3}=1}^{s_{3}} \beta_{n, i_{3}}^{2} a^{i_{3}}\right)\left(\sum_{i_{4}=1}^{s_{4}} \beta_{n, i_{4}}^{3} a^{i_{4}}\right)\left\|y_{n}^{4}-q\right\| .
\end{aligned}
$$

Continuing the above process we have

$$
\begin{aligned}
\left\|x_{n+1}-q\right\| & \left\{\alpha_{n, 0}+\left(\sum_{i_{1}=1}^{s_{1}} \alpha_{n, i_{1}} a^{i_{1}}\right) \beta_{n, 0}^{1}+\left(\sum_{i_{1}=1}^{s_{1}} \alpha_{n, i_{1}} a^{i_{1}}\right)\right. \\
& \times\left(\sum_{i_{2}=1}^{s_{2}} \beta_{n, i_{2}}^{1} a^{i_{2}}\right) \beta_{n, 0}^{2}+\cdots+\left(\sum_{i_{1}=1}^{s_{1}} \alpha_{n, i_{1}} a^{i_{1}}\right) \\
& \left.\times\left(\sum_{i_{2}=1}^{s_{2}} \beta_{n, i_{2}}^{1} a^{i_{2}}\right) \cdots\left(\sum_{i_{k-2}=1}^{s_{k-2}} \beta_{n, i_{k-2}}^{k-3} a^{i_{k-2}}\right) \beta_{n, 0}^{k-2}\right\} \\
\times & \left\|x_{n}-q\right\|+\left(\sum_{i_{1}=1}^{s_{1}} \alpha_{n, i_{1}} a^{i_{1}}\right)\left(\sum_{i_{2}=1}^{s_{2}} \beta_{n, i_{2}}^{1} a^{i_{2}}\right) \cdots \\
& \left(\sum_{i_{k-1}=1}^{s_{k-1}} \beta_{n, i_{k-1}}^{k-2} a^{i_{k-1}}\right)\left\|y_{n}^{k-1}-q\right\| .
\end{aligned}
$$


Using again (4) and Lemma 4, we get

$$
\begin{aligned}
& \left\|y_{n}^{k-1}-q\right\| \\
& =\left\|\beta_{n, 0}^{k-1}\left(x_{n}-q\right)+\sum_{i_{k}=1}^{s_{k}} \beta_{n, i_{k}}^{k-1}\left(T^{i_{k}} x_{n}-T^{i_{k}} q\right)\right\| \\
& \leq \beta_{n, 0}^{k-1}\left\|x_{n}-q\right\|+\sum_{i_{k}=1}^{s_{k}} \beta_{n, i_{k}}^{k-1}\left\|T^{i_{k}} x_{n}-T^{i_{k}} q\right\| \\
& \leq \beta_{n, 0}^{k-1}\left\|x_{n}-q\right\| \\
& +\sum_{i_{k}=1}^{s_{k}} \beta_{n, i_{k}}^{k-1}\left\{\sum_{j=1}^{i_{k}}\left(\begin{array}{c}
i_{k} \\
j
\end{array}\right) a^{i_{k}-j} \varphi^{j}(\|q-T q\|)\right. \\
& \left.+a^{i_{k}}\left\|x_{n}-q\right\|\right\} \\
& =\beta_{n, 0}^{k-1}\left\|x_{n}-q\right\|+\left(\sum_{i_{k}=1}^{s_{k}} \beta_{n, i_{k}}^{k-1} a^{i_{k}}\right)\left\|x_{n}-q\right\| \\
& =\left(\sum_{i_{k}=0}^{s_{k}} \beta_{n, i_{k}}^{k-1} a^{i_{k}}\right)\left\|x_{n}-q\right\| .
\end{aligned}
$$

Substituting (19) into (18) we derive

$$
\begin{aligned}
\| x_{n+1}- & q \| \\
\leq & \left\{\alpha_{n, 0}+\left(\sum_{i_{1}=1}^{s_{1}} \alpha_{n, i_{1}} a^{i_{1}}\right) \beta_{n, 0}^{1}+\left(\sum_{i_{1}=1}^{s_{1}} \alpha_{n, i_{1}} a^{i_{1}}\right)\right. \\
& \times\left(\sum_{i_{2}=1}^{s_{2}} \beta_{n, i_{2}}^{1} a^{i_{2}}\right) \beta_{n, 0}^{2}+\cdots+\left(\sum_{i_{1}=1}^{s_{1}} \alpha_{n, i_{1}} a^{i_{1}}\right) \\
& \times\left(\sum_{i_{2}=1}^{s_{2}} \beta_{n, i_{2}}^{1} a^{i_{2}}\right) \ldots\left(\sum_{i_{k-2}=1}^{s_{k-2}} \beta_{n, i_{k-2}}^{k-3} a^{i_{k-2}}\right) \beta_{n, 0}^{k-2} \\
& +\left(\sum_{i_{1}=1}^{s_{1}} \alpha_{n, i_{1}} a^{i_{1}}\right)\left(\sum_{i_{2}=1}^{s_{2}} \beta_{n, i_{2}}^{1} a^{i_{2}}\right) \ldots \\
& \left.\left(\sum_{i_{k-1}=1}^{s_{k-1}} \beta_{n, i_{k-1}}^{k-2} a^{i_{k-1}}\right)\left(\sum_{i_{k}=0}^{s_{k}} \beta_{n, i_{k}}^{k-1} a^{i_{k}}\right)\right\}\left\|x_{n}-q\right\| .
\end{aligned}
$$

Define

$$
\begin{aligned}
\sigma:=\alpha_{n, 0} & +\left(\sum_{i_{1}=1}^{s_{1}} \alpha_{n, i_{1}} a^{i_{1}}\right) \beta_{n, 0}^{1}+\left(\sum_{i_{1}=1}^{s_{1}} \alpha_{n, i_{1}} a^{i_{1}}\right) \\
& \times\left(\sum_{i_{2}=1}^{s_{2}} \beta_{n, i_{2}}^{1} a^{i_{2}}\right) \beta_{n, 0}^{2}+\cdots+\left(\sum_{i_{1}=1}^{s_{1}} \alpha_{n, i_{1}} a^{i_{1}}\right)
\end{aligned}
$$

$$
\begin{aligned}
\times & \left(\sum_{i_{2}=1}^{s_{2}} \beta_{n, i_{2}}^{1} a^{i_{2}}\right) \cdots\left(\sum_{i_{k-2}=1}^{s_{k-2}} \beta_{n, i_{k-2}}^{k-3} a^{i_{k-2}}\right) \beta_{n, 0}^{k-2} \\
+ & \left(\sum_{i_{1}=1}^{s_{1}} \alpha_{n, i_{1}} a^{i_{1}}\right)\left(\sum_{i_{2}=1}^{s_{2}} \beta_{n, i_{2}}^{1} a^{i_{2}}\right) \cdots \\
& \left(\sum_{i_{k-1}=1}^{s_{k-1}} \beta_{n, i_{k-1}}^{k-2} a^{i_{k-1}}\right)\left(\sum_{i_{k}=0}^{s_{k}} \beta_{n, i_{k}}^{k-1} a^{i_{k}}\right) .
\end{aligned}
$$

Now we show that $\sigma \in[0,1)$. Since $a^{i_{k}} \in[0,1), \alpha_{n, 0}>0$, $\sum_{i_{1}=0}^{s_{1}} \alpha_{n, i_{1}}=1$, and $\sum_{i_{p+1}=0}^{s_{p+1}} \beta_{n, i_{p+1}}^{p}=1$ for $p=\overline{1, k-1}$, we obtain

$$
\begin{aligned}
\sigma< & \alpha_{n, 0}+\left(1-\alpha_{n, 0}\right) \beta_{n, 0}^{1}+\left(1-\alpha_{n, 0}\right)\left(1-\beta_{n, 0}^{1}\right) \beta_{n, 0}^{2} \\
& +\cdots+\left(1-\alpha_{n, 0}\right)\left(1-\beta_{n, 0}^{1}\right) \cdots\left(1-\beta_{n, 0}^{k-3}\right) \beta_{n, 0}^{k-2} \\
& +\left(1-\alpha_{n, 0}\right)\left(1-\beta_{n, 0}^{1}\right) \cdots\left(1-\beta_{n, 0}^{k-3}\right)\left(1-\beta_{n, 0}^{k-2}\right)
\end{aligned}
$$

$=1$.

By an application of Lemma 3 to (20), $\lim _{n \rightarrow \infty} x_{n}=q$.

Theorem 7. Let $x_{0} \in X$ and $\left\{x_{n}\right\}_{n \in \mathbb{N}}$ be a sequence generated by the Kirk multistep iterative scheme (4). Suppose that $T$ has a fixed point $q$. Then the Kirk multistep iterative scheme (4) is T-stable.

Proof. Let $\left\{y_{n}\right\}_{n \in \mathbb{N}} \subset X,\left\{u_{n}^{p}\right\}_{n \in \mathbb{N}}$, for $p=\overline{1, k-1}$, be arbitrary sequences in $X$. Let $\varepsilon_{n}=\left\|y_{n+1}-\alpha_{n, 0} y_{n}-\sum_{i_{1}=1}^{s_{1}} \alpha_{n, i_{1}} T^{i_{1}} u_{n}^{1}\right\|$, $n=0,1,2, \ldots$, where $u_{n}^{p}=\beta_{n, 0}^{p} y_{n}+\sum_{i_{p+1}=1}^{s_{p+1}} \beta_{n, i_{p+1}}^{p} T^{i_{p+1}} u_{n}^{p+1}, p=$ $\overline{1, k-2}, u_{n}^{k-1}=\sum_{i_{k}=0}^{s_{k}} \beta_{n, i_{k}}^{k-1} T^{i_{k}} y_{n}, k \geq 2$ and let $\lim _{n \rightarrow \infty} \varepsilon_{n}=0$. We will prove that $\lim _{n \rightarrow \infty} y_{n}=q$.

It follows from (4) and Lemma 4 that

$$
\begin{gathered}
\left\|y_{n+1}-q\right\|=\| y_{n+1}-\alpha_{n, 0} y_{n}-\sum_{i_{1}=1}^{s_{1}} \alpha_{n, i_{1}} T^{i_{1}} u_{n}^{1}+\alpha_{n, 0} y_{n} \\
\quad+\sum_{i_{1}=1}^{s_{1}} \alpha_{n, i_{1}} T^{i_{1}} u_{n}^{1}-q \| \\
\leq \varepsilon_{n}+\left\|\alpha_{n, 0}\left(y_{n}-q\right)+\sum_{i_{1}=1}^{s_{1}} \alpha_{n, i_{1}}\left(T^{i_{1}} u_{n}^{1}-T^{i_{1}} q\right)\right\| \\
\leq \alpha_{n, 0}\left\|y_{n}-q\right\|+\varepsilon_{n}+\sum_{i_{1}=1}^{s_{1}} \alpha_{n, i_{1}}\left\|T^{i_{1}} u_{n}^{1}-T^{i_{1}} q\right\|
\end{gathered}
$$


6

Abstract and Applied Analysis

$$
\begin{aligned}
& \leq \alpha_{n, 0}\left\|y_{n}-q\right\|+\varepsilon_{n} \\
& +\sum_{i_{1}=1}^{s_{1}} \alpha_{n, i_{1}}\left\{\sum_{j=1}^{i_{1}}\left(\begin{array}{c}
i_{1} \\
j
\end{array}\right) a^{i_{1}-j} \varphi^{j}(\|q-T q\|)\right. \\
& \left.+a^{i_{1}}\left\|u_{n}^{1}-q\right\|\right\} \\
& =\alpha_{n, 0}\left\|y_{n}-q\right\|+\varepsilon_{n}+\left(\sum_{i_{1}=1}^{s_{1}} \alpha_{n, i_{1}} a^{i_{1}}\right)\left\|u_{n}^{1}-q\right\|,
\end{aligned}
$$

$$
\begin{aligned}
& \left\|u_{n}^{1}-q\right\| \\
& =\left\|\beta_{n, 0}^{1}\left(y_{n}-q\right)+\sum_{i_{2}=1}^{s_{2}} \beta_{n, i_{2}}^{1}\left(T^{i_{2}} u_{n}^{2}-T^{i_{2}} q\right)\right\| \\
& \leq \beta_{n, 0}^{1}\left\|y_{n}-q\right\|+\sum_{i_{2}=1}^{s_{2}} \beta_{n, i_{2}}^{1}\left\|T^{i_{2}} u_{n}^{2}-T^{i_{2}} q\right\| \\
& \leq \beta_{n, 0}^{1}\left\|y_{n}-q\right\| \\
& +\sum_{i_{2}=1}^{s_{2}} \beta_{n, i_{2}}^{1}\left\{\sum_{j=1}^{i_{2}}\left(\begin{array}{c}
i_{2} \\
j
\end{array}\right) a^{i_{2}-j} \varphi^{j}(\|q-T q\|)\right. \\
& \left.+a^{i_{2}}\left\|u_{n}^{2}-q\right\|\right\} \\
& =\beta_{n, 0}^{1}\left\|y_{n}-q\right\|+\left(\sum_{i_{2}=1}^{s_{2}} \beta_{n, i_{2}}^{1} a^{i_{2}}\right)\left\|u_{n}^{2}-q\right\|, \\
& \left\|u_{n}^{2}-q\right\| \\
& =\left\|\beta_{n, 0}^{2}\left(y_{n}-q\right)+\sum_{i_{3}=1}^{s_{3}} \beta_{n, i_{3}}^{2}\left(T^{i_{3}} u_{n}^{3}-T^{i_{3}} q\right)\right\| \\
& \leq \beta_{n, 0}^{2}\left\|y_{n}-q\right\|+\sum_{i_{3}=1}^{s_{3}} \beta_{n, i_{3}}^{2}\left\|T^{i_{3}} u_{n}^{3}-T^{i_{3}} q\right\| \\
& \leq \beta_{n, 0}^{2}\left\|y_{n}-q\right\| \\
& +\sum_{i_{3}=1}^{s_{3}} \beta_{n, i_{3}}^{2}\left\{\sum_{j=1}^{i_{3}}\left(\begin{array}{c}
i_{3} \\
j
\end{array}\right) a^{i_{3}-j} \varphi^{j}(\|q-T q\|)\right. \\
& \left.+a^{i_{3}}\left\|u_{n}^{3}-q\right\|\right\} \\
& =\beta_{n, 0}^{2}\left\|y_{n}-q\right\|+\left(\sum_{i_{3}=1}^{s_{3}} \beta_{n, i_{3}}^{2} a^{i_{3}}\right)\left\|u_{n}^{3}-q\right\| .
\end{aligned}
$$

Combining (23), (24), and (25) we have

$$
\begin{aligned}
& \left\|y_{n+1}-q\right\| \\
& \leq\left(\sum_{i_{1}=1}^{s_{1}} \alpha_{n, i_{1}} a^{i_{1}}\right)\left(\sum_{i_{2}=1}^{s_{2}} \beta_{n, i_{2}}^{1} a^{i_{2}}\right)\left(\sum_{i_{3}=1}^{s_{3}} \beta_{n, i_{3}}^{2} a^{i_{3}}\right)\left\|u_{n}^{3}-q\right\| \\
& \quad+\left(\sum_{i_{1}=1}^{s_{1}} \alpha_{n, i_{1}} a^{i_{1}}\right)\left(\sum_{i_{2}=1}^{s_{2}} \beta_{n, i_{2}}^{1} a^{i_{2}}\right) \beta_{n, 0}^{2}\left\|y_{n}-q\right\| \\
& \quad+\left(\sum_{i_{1}=1}^{s_{1}} \alpha_{n, i_{1}} a^{i_{1}}\right) \beta_{n, 0}^{1}\left\|y_{n}-q\right\|+\alpha_{n, 0}\left\|y_{n}-q\right\|+\varepsilon_{n} .
\end{aligned}
$$

By induction we get

$$
\begin{aligned}
& \left\|y_{n+1}-q\right\| \\
& \leq\left(\sum_{i_{1}=1}^{s_{1}} \alpha_{n, i_{1}} a^{i_{1}}\right)\left(\sum_{i_{2}=1}^{s_{2}} \beta_{n, i_{2}}^{1} a^{i_{2}}\right) \ldots \\
& \left(\sum_{i_{k-1}=1}^{s_{k-1}} \beta_{n, i_{k-1}}^{k-2} a^{i_{k-1}}\right)\left\|u_{n}^{k-1}-q\right\| \\
& +\left\{\alpha_{n, 0}+\left(\sum_{i_{1}=1}^{s_{1}} \alpha_{n, i_{1}} a^{i_{1}}\right) \beta_{n, 0}^{1}\right. \\
& +\left(\sum_{i_{1}=1}^{s_{1}} \alpha_{n, i_{1}} a^{i_{1}}\right)\left(\sum_{i_{2}=1}^{s_{2}} \beta_{n, i_{2}}^{1} a^{i_{2}}\right) \beta_{n, 0}^{2} \\
& +\cdots+\left(\sum_{i_{1}=1}^{s_{1}} \alpha_{n, i_{1}} a^{i_{1}}\right)\left(\sum_{i_{2}=1}^{s_{2}} \beta_{n, i_{2}}^{1} a^{i_{2}}\right) \ldots \\
& \left.\left(\sum_{i_{k-2}=1}^{s_{k-2}} \beta_{n, i_{k-2}}^{k-3} a^{i_{k-2}}\right) \beta_{n, 0}^{k-2}\right\}\left\|y_{n}-q\right\|+\varepsilon_{n} .
\end{aligned}
$$

Again using (4) and Lemma 4, we obtain

$$
\begin{aligned}
& \left\|u_{n}^{k-1}-q\right\| \\
& =\left\|\sum_{i_{k}=0}^{s_{k}} \beta_{n, i_{k}}^{k-1} T^{i_{k}} y_{n}-\sum_{i_{k}=0}^{s_{k}} \beta_{n, i_{k}}^{k-1} T^{i_{k}} q\right\| \\
& \leq \beta_{n, 0}^{k-1}\left\|y_{n}-q\right\|+\sum_{i_{k}=1}^{s_{k}} \beta_{n, i_{k}}^{k-1}\left\|T^{i_{k}} y_{n}-T^{i_{k}} q\right\| \\
& \leq \beta_{n, 0}^{k-1}\left\|y_{n}-q\right\|
\end{aligned}
$$




$$
\begin{gathered}
+\sum_{i_{k}=1}^{s_{k}} \beta_{n, i_{k}}^{k-1}\left\{\sum_{j=1}^{i_{k}}\left(\begin{array}{c}
i_{k} \\
j
\end{array}\right) a^{i_{k}-j} \varphi^{j}(\|q-T q\|)\right. \\
\left.+a^{i_{k}}\left\|y_{n}-q\right\|\right\} \\
=\left(\sum_{i_{k}=0}^{s_{k}} \beta_{n, i_{k}}^{k-1} a^{i_{k}}\right)\left\|y_{n}-q\right\| .
\end{gathered}
$$

Substituting (28) into (27) we derive

$$
\begin{aligned}
\left\|y_{n+1}-q\right\| & \left\{\alpha_{n, 0}+\left(\sum_{i_{1}=1}^{s_{1}} \alpha_{n, i_{1}} a^{i_{1}}\right) \beta_{n, 0}^{1}+\left(\sum_{i_{1}=1}^{s_{1}} \alpha_{n, i_{1}} a^{i_{1}}\right)\right. \\
& \times\left(\sum_{i_{2}=1}^{s_{2}} \beta_{n, i_{2}}^{1} a^{i_{2}}\right) \beta_{n, 0}^{2}+\cdots+\left(\sum_{i_{1}=1}^{s_{1}} \alpha_{n, i_{1}} a^{i_{1}}\right) \\
& \times\left(\sum_{i_{2}=1}^{s_{2}} \beta_{n, i_{2}}^{1} a^{i_{2}}\right) \ldots\left(\sum_{i_{k-2}=1}^{s_{k-2}} \beta_{n, i_{k-2}}^{k-3} a^{i_{k-2}}\right) \beta_{n, 0}^{k-2} \\
& +\left(\sum_{i_{1}=1}^{s_{1}} \alpha_{n, i_{1}} a^{i_{1}}\right)\left(\sum_{i_{2}=1}^{s_{2}} \beta_{n, i_{2}}^{1} a^{i_{2}}\right) \ldots \\
& \left.\left(\sum_{i_{k-1}=1}^{s_{k-1}} \beta_{n, i_{k-1}}^{k-2} a^{i_{k-1}}\right)\left(\sum_{i_{k}=0}^{s_{k}} \beta_{n, i_{k}}^{k-1} a^{i_{k}}\right)\right\}
\end{aligned}
$$$$
\times\left\|y_{n}-q\right\|+\varepsilon_{n}
$$

Define

$$
\begin{aligned}
\sigma:= & \alpha_{n, 0}+\left(\sum_{i_{1}=1}^{s_{1}} \alpha_{n, i_{1}} a^{i_{1}}\right) \beta_{n, 0}^{1}+\left(\sum_{i_{1}=1}^{s_{1}} \alpha_{n, i_{1}} a^{i_{1}}\right) \\
& \times\left(\sum_{i_{2}=1}^{s_{2}} \beta_{n, i_{2}}^{1} a^{i_{2}}\right) \beta_{n, 0}^{2}+\cdots+\left(\sum_{i_{1}=1}^{s_{1}} \alpha_{n, i_{1}} a^{i_{1}}\right) \\
& \times\left(\sum_{i_{2}=1}^{s_{2}} \beta_{n, i_{2}}^{1} a^{i_{2}}\right) \cdots\left(\sum_{i_{k-2}=1}^{s_{k-2}} \beta_{n, i_{k-2}}^{k-3} a^{i_{k-2}}\right) \beta_{n, 0}^{k-2}
\end{aligned}
$$

$$
\begin{aligned}
+ & \left(\sum_{i_{1}=1}^{s_{1}} \alpha_{n, i_{1}} a^{i_{1}}\right)\left(\sum_{i_{2}=1}^{s_{2}} \beta_{n, i_{2}}^{1} a^{i_{2}}\right) \cdots \\
& \left(\sum_{i_{k-1}=1}^{s_{k-1}} \beta_{n, i_{k-1}}^{k-2} a^{i_{k-1}}\right)\left(\sum_{i_{k}=0}^{s_{k}} \beta_{n, i_{k}}^{k-1} a^{i_{k}}\right) .
\end{aligned}
$$

We now show that $\sigma \in(0,1)$. Since $a^{i_{k}} \in[0,1), \alpha_{n, 0}>0$, $\sum_{i_{1}=0}^{s_{1}} \alpha_{n, i_{1}}=1$, and $\sum_{i_{p+1}=0}^{s_{p+1}} \beta_{n, i_{p+1}}^{p}=1$ for $p=\overline{1, k-1}$, we have

$$
\begin{aligned}
\sigma< & \alpha_{n, 0}+\left(1-\alpha_{n, 0}\right) \beta_{n, 0}^{1}+\left(1-\alpha_{n, 0}\right)\left(1-\beta_{n, 0}^{1}\right) \beta_{n, 0}^{2} \\
& +\cdots+\left(1-\alpha_{n, 0}\right)\left(1-\beta_{n, 0}^{1}\right) \cdots\left(1-\beta_{n, 0}^{k-3}\right) \beta_{n, 0}^{k-2} \\
& +\left(1-\alpha_{n, 0}\right)\left(1-\beta_{n, 0}^{1}\right) \cdots\left(1-\beta_{n, 0}^{k-2}\right) \\
= & 1
\end{aligned}
$$

that is, $\sigma \in(0,1)$. Therefore, an application of Lemma 4 to (29) yields $\lim _{n \rightarrow \infty} y_{n}=q$.

Now suppose that $\lim _{n \rightarrow \infty} y_{n}=q$. Then we will show that $\lim _{n \rightarrow \infty} \varepsilon_{n}=0$.

Using Lemma 4 we have

$$
\begin{aligned}
\varepsilon_{n} & =\left\|y_{n+1}-\alpha_{n, 0} y_{n}-\sum_{i_{1}=1}^{s_{1}} \alpha_{n, i_{1}} T^{i_{1}} u_{n}^{1}\right\| \\
& \leq\left\|y_{n+1}-q\right\|+\left\|q-\alpha_{n, 0} y_{n}-\sum_{i_{1}=1}^{s_{1}} \alpha_{n, i_{1}} T^{i_{1}} u_{n}^{1}\right\| \\
& =\left\|y_{n+1}-q\right\|+\left\|\alpha_{n, 0}\left(q-y_{n}\right)+\sum_{i_{1}=1}^{s_{1}} \alpha_{n, i_{1}}\left(T^{i_{1}} q-T^{i_{1}} u_{n}^{1}\right)\right\| \\
& \leq\left\|y_{n+1}-q\right\|+\alpha_{n, 0}\left\|y_{n}-q\right\|+\sum_{i_{1}=1}^{s_{1}} \alpha_{n, i_{1}}\left\|T^{i_{1}} q-T^{i_{1}} u_{n}^{1}\right\|
\end{aligned}
$$

$$
\begin{aligned}
& \leq\left\|y_{n+1}-q\right\|+\alpha_{n, 0}\left\|y_{n}-q\right\| \\
&+ \sum_{i_{1}=1}^{s_{1}} \alpha_{n, i_{1}}\left\{\sum_{j=1}^{i_{1}}\left(\begin{array}{c}
i_{1} \\
j
\end{array}\right) a^{i_{1}-j} \varphi^{j}(\|q-T q\|)\right. \\
&\left.+a^{i_{1}}\left\|q-u_{n}^{1}\right\|\right\} \\
& \leq\left\|y_{n+1}-q\right\|+\alpha_{n, 0}\left\|y_{n}-q\right\|+\left(\sum_{i_{1}=1}^{s_{1}} \alpha_{n, i_{1}} a^{i_{1}}\right)\left\|q-u_{n}^{1}\right\|,
\end{aligned}
$$


8

Abstract and Applied Analysis

$$
\begin{aligned}
& \left\|q-u_{n}^{1}\right\| \\
& =\left\|q-\beta_{n, 0}^{1} y_{n}-\sum_{i_{2}=1}^{s_{2}} \beta_{n, i_{2}}^{1} T^{i_{2}} u_{n}^{2}\right\| \\
& =\left\|\beta_{n, 0}^{1}\left(q-y_{n}\right)+\sum_{i_{2}=1}^{s_{2}} \beta_{n, i_{2}}^{1}\left(T^{i_{2}} q-T^{i_{2}} u_{n}^{2}\right)\right\| \\
& \leq \beta_{n, 0}^{1}\left\|y_{n}-q\right\|+\sum_{i_{2}=1}^{s_{2}} \beta_{n, i_{2}}^{1}\left\|T^{i_{2}} q-T^{i_{2}} u_{n}^{2}\right\| \\
& \leq \beta_{n, 0}^{1}\left\|y_{n}-q\right\| \\
& +\sum_{i_{2}=1}^{s_{2}} \beta_{n, i_{2}}^{1}\left\{\sum_{j=1}^{i_{2}}\left(\begin{array}{c}
i_{2} \\
j
\end{array}\right) a^{i_{2}-j} \varphi^{j}(\|q-T q\|)\right. \\
& \left.+a^{i_{2}}\left\|q-u_{n}^{2}\right\|\right\} \\
& \leq \beta_{n, 0}^{1}\left\|y_{n}-q\right\|+\left(\sum_{i_{2}=1}^{s_{2}} \beta_{n, i_{2}}^{1} a^{i_{2}}\right)\left\|q-u_{n}^{2}\right\|, \\
& \left\|q-u_{n}^{2}\right\| \\
& =\left\|q-\beta_{n, 0}^{2} y_{n}-\sum_{i_{3}=1}^{s_{3}} \beta_{n, i_{3}}^{2} T^{i_{3}} u_{n}^{3}\right\| \\
& =\left\|\beta_{n, 0}^{2}\left(q-y_{n}\right)+\sum_{i_{3}=1}^{s_{3}} \beta_{n, i_{3}}^{2}\left(T^{i_{3}} q-T^{i_{3}} u_{n}^{3}\right)\right\| \\
& \leq \beta_{n, 0}^{2}\left\|y_{n}-q\right\|+\sum_{i_{3}=1}^{s_{3}} \beta_{n, i_{3}}^{2}\left\|T^{i_{3}} q-T^{i_{3}} u_{n}^{3}\right\| \\
& \leq \beta_{n, 0}^{2}\left\|y_{n}-q\right\| \\
& +\sum_{i_{3}=1}^{s_{3}} \beta_{n, i_{3}}^{2}\left\{\sum_{j=1}^{i_{3}}\left(\begin{array}{c}
i_{3} \\
j
\end{array}\right) a^{i_{3}-j} \varphi^{j}(\|q-T q\|)\right. \\
& \left.+a^{i_{3}}\left\|q-u_{n}^{3}\right\|\right\} \\
& \leq \beta_{n, 0}^{2}\left\|y_{n}-q\right\|+\left(\sum_{i_{3}=1}^{s_{3}} \beta_{n, i_{3}}^{2} a^{i_{3}}\right)\left\|q-u_{n}^{3}\right\| .
\end{aligned}
$$

Thus, by induction, we get

$$
\begin{aligned}
\varepsilon_{n} \leq & \left\|y_{n+1}-q\right\|+\alpha_{n, 0}\left\|y_{n}-q\right\|+\left(\sum_{i_{1}=1}^{s_{1}} \alpha_{n, i_{1}} a^{i_{1}}\right) \\
& \times\left(\sum_{i_{2}=1}^{s_{2}} \beta_{n, i_{2}}^{1} a^{i_{2}}\right) \ldots\left(\sum_{i_{k-1}=1}^{s_{k-1}} \beta_{n, i_{k-1}}^{k-2} a^{i_{k-1}}\right) \\
& \times\left\|q-u_{n}^{k-1}\right\|+\left(\sum_{i_{1}=1}^{s_{1}} \alpha_{n, i_{1}} a^{i_{1}}\right) \\
& \times\left(\sum_{i_{2}=1}^{s_{2}} \beta_{n, i_{2}}^{1} a^{i_{2}}\right) \ldots\left(\sum_{i_{k-2}=1}^{s_{k-2}} \beta_{n, i_{k-2}}^{k-3} a^{i_{k-2}}\right) \beta_{n, 0}^{k-2} \\
& \times\left\|y_{n}-q\right\|+\cdots+\left(\sum_{i_{1}=1}^{s_{1}} \alpha_{n, i_{1}} a^{i_{1}}\right)\left(\sum_{i_{2}=1}^{s_{2}} \beta_{n, i_{2}}^{1} a^{i_{2}}\right) \beta_{n, 0}^{2} \\
& \times\left\|y_{n}-q\right\|+\left(\sum_{i_{1}=1}^{s_{1}} \alpha_{n, i_{1}} a^{i_{1}}\right) \beta_{n, 0}^{1}\left\|y_{n}-q\right\| .
\end{aligned}
$$

It follows from the relation (32), (33), and (34) that

$$
\begin{aligned}
\varepsilon_{n} \leq & \left\|y_{n+1}-q\right\|+\alpha_{n, 0}\left\|y_{n}-q\right\| \\
& +\left(\sum_{i_{1}=1}^{s_{1}} \alpha_{n, i_{1}} a^{i_{1}}\right)\left(\sum_{i_{2}=1}^{s_{2}} \beta_{n, i_{2}}^{1} a^{i_{2}}\right)\left(\sum_{i_{3}=1}^{s_{3}} \beta_{n, i_{3}}^{2} a^{i_{3}}\right)\left\|q-u_{n}^{3}\right\|
\end{aligned}
$$

(36)

Utilizing (4) and Lemma 4, we obtain

$$
\begin{aligned}
& \left\|q-u_{n}^{k-1}\right\| \\
& =\left\|\sum_{i_{k}=0}^{s_{k}} \beta_{n, i_{k}}^{k-1} T^{i_{k}} q-\sum_{i_{k}=0}^{s_{k}} \beta_{n, i_{k}}^{k-1} T^{i_{k}} y_{n}\right\| \\
& \leq \beta_{n, 0}^{k-1}\left\|y_{n}-q\right\|+\sum_{i_{k}=1}^{s_{k}} \beta_{n, i_{k}}^{k-1}\left\|T^{i_{k}} q-T^{i_{k}} y_{n}\right\| \\
& \leq \beta_{n, 0}^{k-1}\left\|y_{n}-q\right\| \\
& \quad+\sum_{i_{k}=1}^{s_{k}} \beta_{n, i_{k}}^{k-1}\left\{\sum_{j=1}^{i_{k}}\left(\begin{array}{c}
i_{k} \\
j
\end{array}\right) a^{i_{k}-j} \varphi^{j}(\|q-T q\|)\right. \\
& \left.\quad+a^{i_{k}}\left\|y_{n}-q\right\|\right\} \\
& =\left(\sum_{i_{k}=0}^{s_{k}} \beta_{n, i_{k}}^{k-1} a^{i_{k}}\right)\left\|y_{n}-q\right\| .
\end{aligned}
$$


Substituting (37) into (36) gives

$$
\begin{aligned}
\varepsilon_{n} \leq & \left\|y_{n+1}-q\right\| \\
& +\left\{\alpha_{n, 0}+\left(\sum_{i_{1}=1}^{s_{1}} \alpha_{n, i_{1}} a^{i_{1}}\right) \beta_{n, 0}^{1}+\left(\sum_{i_{1}=1}^{s_{1}} \alpha_{n, i_{1}} a^{i_{1}}\right)\right. \\
& \times\left(\sum_{i_{2}=1}^{s_{2}} \beta_{n, i_{2}}^{1} a^{i_{2}}\right) \beta_{n, 0}^{2}+\cdots+\left(\sum_{i_{1}=1}^{s_{1}} \alpha_{n, i_{1}} a^{i_{1}}\right) \\
& \times\left(\sum_{i_{2}=1}^{s_{2}} \beta_{n, i_{2}}^{1} a^{i_{2}}\right) \cdots\left(\sum_{i_{k-2}=1}^{s_{k-2}} \beta_{n, i_{k-2}}^{k-3} a^{i_{k-2}}\right) \beta_{n, 0}^{k-2} \\
+ & \left(\sum_{i_{1}=1}^{s_{1}} \alpha_{n, i_{1}} a^{i_{1}}\right)\left(\sum_{i_{2}=1}^{s_{2}} \beta_{n, i_{2}}^{1} a^{i_{2}}\right) \ldots \\
& \left.\left(\sum_{i_{k-1}=1}^{s_{k-1}} \beta_{n, i_{k-1}}^{k-2} a^{i_{k-1}}\right)\left(\sum_{i_{k}=0}^{s_{k}} \beta_{n, i_{k}}^{k-1} a^{i_{k}}\right)\right\}\left\|y_{n}-q\right\| .
\end{aligned}
$$

Again define

$$
\begin{aligned}
\sigma:= & \alpha_{n, 0}+\left(\sum_{i_{1}=1}^{s_{1}} \alpha_{n, i_{1}} a^{i_{1}}\right) \beta_{n, 0}^{1}+\left(\sum_{i_{1}=1}^{s_{1}} \alpha_{n, i_{1}} a^{i_{1}}\right) \\
& \times\left(\sum_{i_{2}=1}^{s_{2}} \beta_{n, i_{2}}^{1} a^{i_{2}}\right) \beta_{n, 0}^{2}+\cdots+\left(\sum_{i_{1}=1}^{s_{1}} \alpha_{n, i_{1}} a^{i_{1}}\right) \\
& \times\left(\sum_{i_{2}=1}^{s_{2}} \beta_{n, i_{2}}^{1} a^{i_{2}}\right) \cdots\left(\sum_{i_{k-2}=1}^{s_{k-2}} \beta_{n, i_{k-2}}^{k-3} a^{i_{k-2}}\right) \beta_{n, 0}^{k-2} \\
& +\left(\sum_{i_{1}=1}^{s_{1}} \alpha_{n, i_{1}} a^{i_{1}}\right)\left(\sum_{i_{2}=1}^{s_{2}} \beta_{n, i_{2}}^{1} a^{i_{2}}\right) \cdots \\
& \left(\sum_{i_{k-1}=1}^{s_{k-1}} \beta_{n, i_{k-1}}^{k-2} a^{i_{k-1}}\right)\left(\sum_{i_{k}=0}^{s_{k}} \beta_{n, i_{k}}^{k-1} a^{i_{k}}\right) .
\end{aligned}
$$

Hence (38) becomes

$$
\varepsilon_{n} \leq\left\|y_{n+1}-q\right\|+\sigma\left\|y_{n}-q\right\|
$$

Using same argument as that of first part of the proof we obtain $\sigma \in(0,1)$.

It therefore follows from assumption $\lim _{n \rightarrow \infty} y_{n}=q$ that $\varepsilon_{n} \rightarrow 0$ as $n \rightarrow \infty$.

Theorem 8. Let $x_{0} \in X$ and $\left\{x_{n}\right\}_{n \in \mathbb{N}}$ be a sequence generated by the Kirk-SP iterative scheme (3). Suppose that $T$ has a fixed point q. Then the Kirk-SP iterative scheme (3) is T-stable.

Proof. Let $\left\{y_{n}\right\}_{n \in \mathbb{N}} \subset X, \varepsilon_{n}=\left\|y_{n+1}-\sum_{i_{1}=0}^{s_{1}} \alpha_{n, i_{1}} T^{i_{1}} u_{n}^{1}\right\|, n=$ $0,1,2, \ldots, u_{n}^{1}=\sum_{i_{2}=0}^{s_{2}} \beta_{n, i_{2}}^{1} T^{i_{2}} u_{n}^{2}$, and $u_{n}^{2}=\sum_{i_{3}=0}^{s_{3}} \beta_{n, i_{3}}^{2} T^{i_{3}} y_{n}$. Assume that $\lim _{n \rightarrow \infty} \varepsilon_{n}=0$. We will prove that $\lim _{n \rightarrow \infty} y_{n}=$ $q$.
It follows from (3) and Lemma 4 that

$$
\begin{aligned}
& \left\|y_{n+1}-q\right\| \\
& =\left\|y_{n+1}-\sum_{i_{1}=0}^{s_{1}} \alpha_{n, i_{1}} T^{i_{1}} u_{n}^{1}+\sum_{i_{1}=0}^{s_{1}} \alpha_{n, i_{1}} T^{i_{1}} u_{n}^{1}-q\right\| \\
& \leq \varepsilon_{n}+\left\|\sum_{i_{1}=0}^{s_{1}} \alpha_{n, i_{1}}\left(T^{i_{1}} u_{n}^{1}-T^{i_{1}} q\right)\right\| \\
& \leq \varepsilon_{n}+\alpha_{n, 0}\left\|u_{n}^{1}-q\right\|+\sum_{i_{1}=1}^{s_{1}} \alpha_{n, i_{1}}\left\|T^{i_{1}} u_{n}^{1}-T^{i_{1}} q\right\| \\
& \leq \varepsilon_{n}+\alpha_{n, 0}\left\|u_{n}^{1}-q\right\| \\
& +\sum_{i_{1}=1}^{s_{1}} \alpha_{n, i_{1}}\left\{\sum_{j=1}^{i_{1}}\left(\begin{array}{c}
i_{1} \\
j
\end{array}\right) a^{i_{1}-j} \varphi^{j}(\|q-T q\|)\right. \\
& \left.+a^{i_{1}}\left\|u_{n}^{1}-q\right\|\right\} \\
& =\varepsilon_{n}+\left(\sum_{i_{1}=0}^{s_{1}} \alpha_{n, i_{1}} a^{i_{1}}\right)\left\|u_{n}^{1}-q\right\|, \\
& \left\|u_{n}^{1}-q\right\| \\
& =\left\|\sum_{i_{2}=0}^{s_{2}} \beta_{n, i_{2}}^{1}\left(T^{i_{2}} u_{n}^{2}-T^{i_{2}} q\right)\right\| \\
& \leq \beta_{n, 0}^{1}\left\|u_{n}^{2}-q\right\|+\sum_{i_{2}=1}^{s_{2}} \beta_{n, i_{2}}^{1}\left\|T^{i_{2}} u_{n}^{2}-T^{i_{2}} q\right\| \\
& \leq \beta_{n, 0}^{1}\left\|u_{n}^{2}-q\right\| \\
& +\sum_{i_{2}=1}^{s_{2}} \beta_{n, i_{2}}^{1}\left\{\sum_{j=1}^{i_{2}}\left(\begin{array}{c}
i_{2} \\
j
\end{array}\right) a^{i_{2}-j} \varphi^{j}(\|q-T q\|)\right. \\
& \left.+a^{i_{2}}\left\|u_{n}^{2}-q\right\|\right\} \\
& =\left(\sum_{i_{2}=0}^{s_{2}} \beta_{n, i_{2}}^{1} a^{i_{2}}\right)\left\|u_{n}^{2}-q\right\|, \\
& \left\|u_{n}^{2}-q\right\| \\
& =\left\|\sum_{i_{3}=0}^{s_{3}} \beta_{n, i_{3}}^{2}\left(T^{i_{3}} y_{n}-T^{i_{3}} q\right)\right\| \\
& \leq \beta_{n, 0}^{2}\left\|y_{n}-q\right\|+\sum_{i_{3}=1}^{s_{3}} \beta_{n, i_{3}}^{2}\left\|T^{i_{3}} y_{n}-T^{i_{3}} q\right\| \\
& \leq \beta_{n, 0}^{2}\left\|y_{n}-q\right\|
\end{aligned}
$$




$$
\begin{gathered}
+\sum_{i_{3}=1}^{s_{3}} \beta_{n, i_{3}}^{2}\left\{\sum_{j=1}^{i_{3}}\left(\begin{array}{c}
i_{3} \\
j
\end{array}\right) a^{i_{3}-j} \varphi^{j}(\|q-T q\|)\right. \\
\left.+a^{i_{3}}\left\|y_{n}-q\right\|\right\} \\
=\left(\sum_{i_{3}=0}^{s_{3}} \beta_{n, i_{3}}^{2} a^{i_{3}}\right)\left\|y_{n}-q\right\| .
\end{gathered}
$$

Combining (41), (42), and (43) we get

$$
\begin{aligned}
\left\|y_{n+1}-q\right\| \leq & \varepsilon_{n}+\left(\sum_{i_{1}=0}^{s_{1}} \alpha_{n, i_{1}} a^{i_{1}}\right)\left(\sum_{i_{2}=0}^{s_{2}} \beta_{n, i_{2}}^{1} a^{i_{2}}\right) \\
& \times\left(\sum_{i_{3}=0}^{s_{3}} \beta_{n, i_{3}}^{2} a^{i_{3}}\right)\left\|y_{n}-q\right\| .
\end{aligned}
$$

Define

$$
\sigma:=\left(\sum_{i_{1}=0}^{s_{1}} \alpha_{n, i_{1}} a^{i_{1}}\right)\left(\sum_{i_{2}=0}^{s_{2}} \beta_{n, i_{2}}^{1} a^{i_{2}}\right)\left(\sum_{i_{3}=0}^{s_{3}} \beta_{n, i_{3}}^{2} a^{i_{3}}\right) .
$$

Thus we can rewrite (44) as follows:

$$
\left\|y_{n+1}-q\right\| \leq \sigma\left\|y_{n}-q\right\|+\varepsilon_{n}
$$

We now show that $\sigma \in(0,1)$. Since $a^{i_{k}} \in[0,1), \alpha_{n, 0}>0$, $\sum_{i_{1}=0}^{s_{1}} \alpha_{n, i_{1}}=1$, and $\sum_{i_{p+1}=0}^{s_{p+1}} \beta_{n, i_{p+1}}^{p}=1$ for $p=\overline{1, k-1}$,

$$
\sigma<\left(\sum_{i_{1}=0}^{s_{1}} \alpha_{n, i_{1}}\right)\left(\sum_{i_{2}=0}^{s_{2}} \beta_{n, i_{2}}^{1}\right)\left(\sum_{i_{3}=0}^{s_{3}} \beta_{n, i_{3}}^{2}\right)=1 .
$$

Therefore, an application of Lemma 3 to (46) yields $\lim _{n \rightarrow \infty} y_{n}=q$.

Now suppose that $\lim _{n \rightarrow \infty} y_{n}=q$. Then we will show that $\lim _{n \rightarrow \infty} \varepsilon_{n}=0$.

Using Lemma 4 we have

$\varepsilon_{n}$

$$
=\left\|y_{n+1}-\sum_{i_{1}=0}^{s_{1}} \alpha_{n, i_{1}} T^{i_{1}} u_{n}^{1}\right\|
$$

$$
\begin{aligned}
& \leq\left\|y_{n+1}-q\right\|+\left\|\sum_{i_{1}=0}^{s_{1}} \alpha_{n, i_{1}}\left(T^{i_{1}} q-T^{i_{1}} u_{n}^{1}\right)\right\| \\
& \leq\left\|y_{n+1}-q\right\|+\alpha_{n, 0}\left\|q-u_{n}^{1}\right\| \\
& +\sum_{i_{1}=1}^{s_{1}} \alpha_{n, i_{1}}\left\|T^{i_{1}} q-T^{i_{1}} u_{n}^{1}\right\| \\
& \leq\left\|y_{n+1}-q\right\|+\alpha_{n, 0}\left\|q-u_{n}^{1}\right\| \\
& +\sum_{i_{1}=1}^{s_{1}} \alpha_{n, i_{1}}\left\{\sum_{j=1}^{i_{1}}\left(\begin{array}{c}
i_{1} \\
j
\end{array}\right) a^{i_{1}-j} \varphi^{j}(\|q-T q\|)\right. \\
& \left.+a^{i_{1}}\left\|q-u_{n}^{1}\right\|\right\} \\
& \leq\left\|y_{n+1}-q\right\|+\left(\sum_{i_{1}=0}^{s_{1}} \alpha_{n, i_{1}} a^{i_{1}}\right)\left\|q-u_{n}^{1}\right\|,
\end{aligned}
$$

$$
\begin{aligned}
\| q & -u_{n}^{1} \| \\
= & \left\|\sum_{i_{2}=0}^{s_{2}} \beta_{n, i_{2}}^{1}\left(T^{i_{2}} q-T^{i_{2}} u_{n}^{2}\right)\right\| \\
\leq & \beta_{n, 0}^{1}\left\|q-u_{n}^{2}\right\|+\sum_{i_{2}=1}^{s_{2}} \beta_{n, i_{2}}^{1}\left\|T^{i_{2}} q-T^{i_{2}} u_{n}^{2}\right\| \\
\leq & \beta_{n, 0}^{1}\left\|q-u_{n}^{2}\right\| \\
& +\sum_{i_{2}=1}^{s_{2}} \beta_{n, i_{2}}^{1}\left\{\sum_{j=1}^{i_{2}}\left(\begin{array}{c}
i_{2} \\
j
\end{array}\right) a^{i_{2}-j} \varphi^{j}(\|q-T q\|)\right. \\
\leq & \left(\sum_{i_{2}=0}^{s_{2}} \beta_{n, i_{2}}^{1} a^{i_{2}}\right)\left\|q-u_{n}^{2}\right\|,
\end{aligned}
$$

$\left\|q-u_{n}^{2}\right\|$

$$
\begin{aligned}
& =\left\|\sum_{i_{3}=0}^{s_{3}} \beta_{n, i_{3}}^{2}\left(T^{i_{3}} q-T^{i_{3}} y_{n}\right)\right\| \\
& \leq \beta_{n, 0}^{2}\left\|q-y_{n}\right\|+\sum_{i_{3}=1}^{s_{3}} \beta_{n, i_{3}}^{2}\left\|T^{i_{3}} q-T^{i_{3}} y_{n}\right\| \\
& \leq \beta_{n, 0}^{2}\left\|q-y_{n}\right\|
\end{aligned}
$$




$$
\begin{gathered}
+\sum_{i_{3}=1}^{s_{3}} \beta_{n, i_{3}}^{2}\left\{\sum_{j=1}^{i_{3}}\left(\begin{array}{c}
i_{3} \\
j
\end{array}\right) a^{i_{3}-j} \varphi^{j}(\|q-T q\|)\right. \\
\left.+a^{i_{3}}\left\|q-y_{n}\right\|\right\} \\
=\left(\sum_{i_{3}=0}^{s_{3}} \beta_{n, i_{3}}^{2} a^{i_{3}}\right)\left\|y_{n}-q\right\| .
\end{gathered}
$$

It follows from (48), (49), and (50) that

$$
\begin{aligned}
\varepsilon_{n} \leq & \left\|y_{n+1}-q\right\| \\
& +\left(\sum_{i_{1}=0}^{s_{1}} \alpha_{n, i_{1}} a^{i_{1}}\right)\left(\sum_{i_{2}=0}^{s_{2}} \beta_{n, i_{2}}^{1} a^{i_{2}}\right)\left(\sum_{i_{3}=0}^{s_{3}} \beta_{n, i_{3}}^{2} a^{i_{3}}\right)\left\|y_{n}-q\right\| .
\end{aligned}
$$

Again define

$$
\sigma:=\left(\sum_{i_{1}=0}^{s_{1}} \alpha_{n, i_{1}} a^{i_{1}}\right)\left(\sum_{i_{2}=0}^{s_{2}} \beta_{n, i_{2}}^{1} a^{i_{2}}\right)\left(\sum_{i_{3}=0}^{s_{3}} \beta_{n, i_{3}}^{2} a^{i_{3}}\right) .
$$

Using same argument as that of first part of the proof we obtain $\sigma \in(0,1)$ and it thus follows from assumption $\lim _{n \rightarrow \infty} y_{n}=q$ that $\varepsilon_{n} \rightarrow 0$ as $n \rightarrow \infty$.

Remark 9. Theorem 6 is a generalization and extension of both Theorems 1 and 2 of Berinde [37], Theorems 2 and 3 of Kannan [38], Theorem 3 of Kannan [39], Theorem 4 of Rhoades [40], Theorem 8 of Rhoades [41], Theorem 2.1 of Olatinwo [42], Theorem 2.6 of Hussain et al. [14], and Theorem 3.1 of Şoltuz and Grosan [43]. Theorem 7 is a generalization and extension of Theorem 2 of Osilike [27], Theorems 2 and 5 of Osilike and Udomene [29] as well as Theorem 3 of Olatinwo et al. [34], Theorems 3.1 and 3.2 of Olatinwo [16], and Theorem 3.1 of Chugh and Kumar [15].

\section{Conflict of Interests}

The authors declare that there is no conflict of interests regarding the publication of this paper.

\section{Acknowledgment}

The first two authors would like to thank Yildiz Technical University Scientific Research Projects Coordination Department under Project no. BAPK 2012-07-03-DOP02 for financial support during the preparation of this paper.

\section{References}

[1] B. E. Rhoades and S. M. Şoltuz, "The equivalence between Mann-Ishikawa iterations and multistep iteration," Nonlinear Analysis: Theory, Methods \& Applications, vol. 58, no. 1-2, pp. 219-228, 2004.
[2] F. Gürsoy, V. Karakaya, and B. E. Rhoades, "Data dependence results of new multistep and Siterative schemes for contractivelike operators," Fixed Point Theory and Applications, vol. 2013, article 76, 2013.

[3] G. Das and J. P. Debata, "Fixed points of quasinonexpansive mappings," Indian Journal of Pure and Applied Mathematics, vol. 17, no. 11, pp. 1263-1269, 1986.

[4] M. A. Noor, "New approximation schemes for general variational inequalities," Journal of Mathematical Analysis and Applications, vol. 251, no. 1, pp. 217-229, 2000.

[5] R. P. Agarwal, D. O'Regan, and D. R. Sahu, "Iterative construction of fixed points of nearly asymptotically nonexpansive mappings," Journal of Nonlinear and Convex Analysis, vol. 8, no. 1, pp. 61-79, 2007.

[6] S. Ishikawa, "Fixed points by a new iteration method," Proceedings of the American Mathematical Society, vol. 44, pp. 147-150, 1974.

[7] S. Thianwan, "Common fixed points of new iterations for two asymptotically nonexpansive nonself-mappings in a Banach space," Journal of Computational and Applied Mathematics, vol. 224, no. 2, pp. 688-695, 2009.

[8] W. Phuengrattana and S. Suantai, "On the rate of convergence of Mann, Ishikawa, Noor and SP-iterations for continuous functions on an arbitrary interval," Journal of Computational and Applied Mathematics, vol. 235, no. 9, pp. 3006-3014, 2011.

[9] W. Takahashi, "Iterative methods for approximation of fixed points and their applications," Journal of the Operations Research Society of Japan, vol. 43, no. 1, pp. 87-108, 2000.

[10] W. R. Mann, "Mean value methods in iteration," Proceedings of the American Mathematical Society, vol. 4, pp. 506-510, 1953.

[11] F. Gürsoy, V. Karakaya, and B. E. Rhoades, "The equivalence among new multistep iteration, siteration and some other iterative schemes," http://arxiv.org/abs/1211.5701v3.

[12] M. A. Krasnoselkij, “Two remarks on the method of successive approximations," Uspekhi Matematicheskikh Nauk, vol. 63, no. 1, pp. 123-127, 1955.

[13] E. Picard, "Memoire sur la theorie des equations aux derivees partielles et la methode des approximations successives," Journal de Mathématiques Pures et Appliquées, vol. 6, pp. 145-210, 1890.

[14] N. Hussain, R. Chugh, V. Kumar, and A. Rafiq, "On the rate of convergence of Kirk-type iterative schemes," Journal of Applied Mathematics, vol. 2012, Article ID 526503, 22 pages, 2012.

[15] R. Chugh and V. Kumar, "Stability of hybrid fixed point iterative algorithms of Kirk-Noor type in normed linear space for self and nonself operators," International Journal of Contemporary Mathematical Sciences, vol. 7, no. 21-24, pp. 1165-1184, 2012.

[16] M. O. Olatinwo, "Some stability results for two hybrid fixed point iterative algorithms in normed linear space," Matematichki Vesnik, vol. 61, no. 4, pp. 247-256, 2009.

[17] W. A. Kirk, "On successive approximations for nonexpansive mappings in Banach spaces," Glasgow Mathematical Journal, vol. 12, pp. 6-9, 1971.

[18] M. Urabe, "Convergence of numerical iteration in solution of equations," Journal of Science of the Hiroshima University A, vol. 19, pp. 479-489, 1956.

[19] A. M. Harder and T. L. Hicks, "A stable iteration procedure for nonexpansive mappings," Mathematica Japonica, vol. 33, no. 5, pp. 687-692, 1988.

[20] A. M. Harder and T. L. Hicks, "Stability results for fixed point iteration procedures," Mathematica Japonica, vol. 33, no. 5, pp. 693-706, 1988. 
[21] A. M. Ostrowski, "The round-off stability of iterations," Zeitschrift für Angewandte Mathematik und Mechanik, vol. 47, pp. 77-81, 1967.

[22] A. M. Harder, Fixed point theory and stability results for fixed points iteration procedures [Ph.D. thesis], University of MissouriRolla, 1987.

[23] B. E. Rhoades, "Fixed point theorems and stability results for fixed point iteration procedures," Indian Journal of Pure and Applied Mathematics, vol. 21, no. 1, pp. 1-9, 1990.

[24] B. E. Rhoades, "Fixed point theorems and stability results for fixed point iteration procedures. II," Indian Journal of Pure and Applied Mathematics, vol. 24, no. 11, pp. 691-703, 1993.

[25] V. Berinde, "Summable almost stability of fixed point iteration procedures," Carpathian Journal of Mathematics, vol. 19, no. 2, pp. 81-88, 2003.

[26] V. Berinde, "On the stability of some fixed point procedures," Buletinul Ştiinţific al Universităţii Baia Mare, vol. 18, no. 1, pp. 7-14, 2002.

[27] M. O. Osilike, "On stability results for fixed point iteration procedures," Journal of the Nigerian Mathematical Society, vol. 14-15, pp. 17-29, 1996.

[28] M. O. Osilike, "Stability results for the Ishikawa fixed point iteration procedure," Indian Journal of Pure and Applied Mathematics, vol. 26, no. 10, pp. 937-945, 1995.

[29] M. O. Osilike and A. Udomene, "Short proofs of stability results for fixed point iteration procedures for a class of contractivetype mappings," Indian Journal of Pure and Applied Mathematics, vol. 30, no. 12, pp. 1229-1234, 1999.

[30] M. O. Olatinwo, "Some stabilty results for two hybrid fixed point iterative algorithms of Kirk-Ishikawa and Kirk-Mann type," Journal of Advanced Mathematical Studies, vol. 1, no. 1-2, pp. 8796, 2008.

[31] M. O. Olatinwo, "Some unifying results on stability and strong convergence for some new iteration processes," Acta Mathematica Academiae Paedagogicae Nyiregyhaziensis, vol. 25, no. 1, pp. 105-118, 2009.

[32] C. O. Imoru and M. O. Olatinwo, "On the stability of Picard and Mann iteration processes," Carpathian Journal of Mathematics, vol. 19, no. 2, pp. 155-160, 2003.

[33] M. O. Olatinwo, O. O. Owojori, and C. O. Imoru, "On some stability results for fixed point iteration procedure," Journal of Mathematics and Statistics, vol. 2, no. 1, pp. 339-342, 2006.

[34] M. O. Olatinwo, O. O. Owojori, and C. O. Imoru, "Some stability results on Krasnolslseskij and Ishikawa fixed point iteration procedures," Journal of Mathematics and Statistics, vol. 2, no. 1, pp. 360-362, 2006.

[35] M. O. Olatinwo, O. O. Owojori, and C. O. Imoru, "Some stability results for fixed point iteration processes," The Australian Journal of Mathematical Analysis and Applications, vol. 3, no. 2, pp. 1-7, 2006.

[36] V. Berinde, Iterative Approximation of Fixed Points, vol. 1912, Springer, Berlin, Germany, 2nd edition, 2007.

[37] V. Berinde, "On the convergence of the Ishikawa iteration in the class of quasi contractive operators," Acta Mathematica Universitatis Comenianae, vol. 73, no. 1, pp. 119-126, 2004.

[38] R. Kannan, "Some results on fixed points," Bulletin of the Calcutta Mathematical Society, vol. 60, pp. 71-76, 1968.

[39] R. Kannan, "Construction of fixed points of a class of nonlinear mappings," Journal of Mathematical Analysis and Applications, vol. 41, pp. 430-438, 1973.
[40] B. E. Rhoades, "Fixed point iterations using infinite matrices," Transactions of the American Mathematical Society, vol. 196, pp. 161-176, 1974.

[41] B. E. Rhoades, "Comments on two fixed point iteration methods," Journal of Mathematical Analysis and Applications, vol. 56, no. 3, pp. 741-750, 1976.

[42] M. O. Olatinwo, "Convergence and stability results for some iterative schemes," Acta Universitatis Apulensis, no. 26, pp. 225236, 2011.

[43] Ş. M. Şoltuz and T. Grosan, "Data dependence for Ishikawa iteration when dealing with contractive-like operators," Fixed Point Theory and Applications, vol. 2008, Article ID 242916, 7 pages, 2008. 


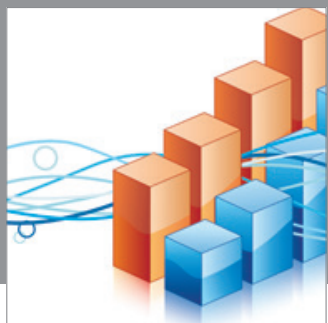

Advances in

Operations Research

mansans

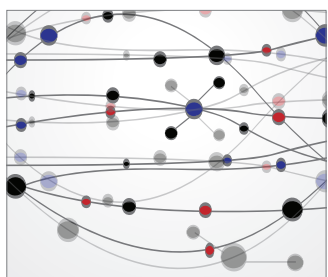

The Scientific World Journal
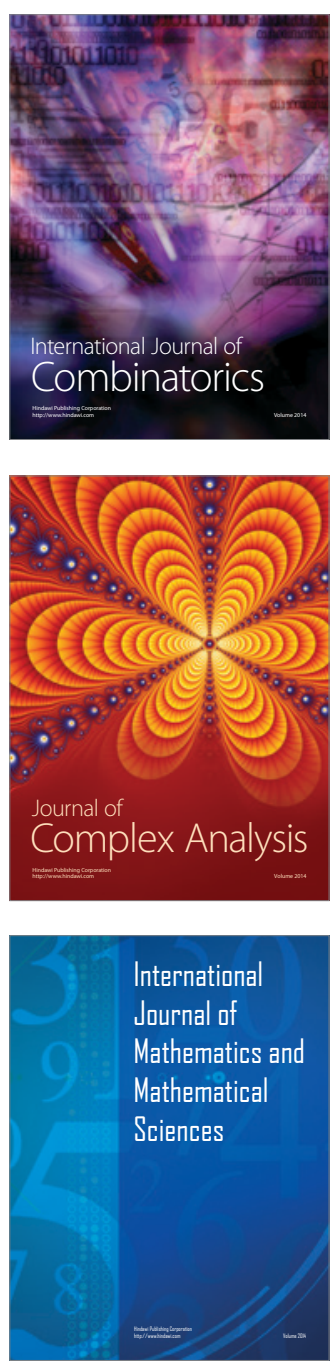
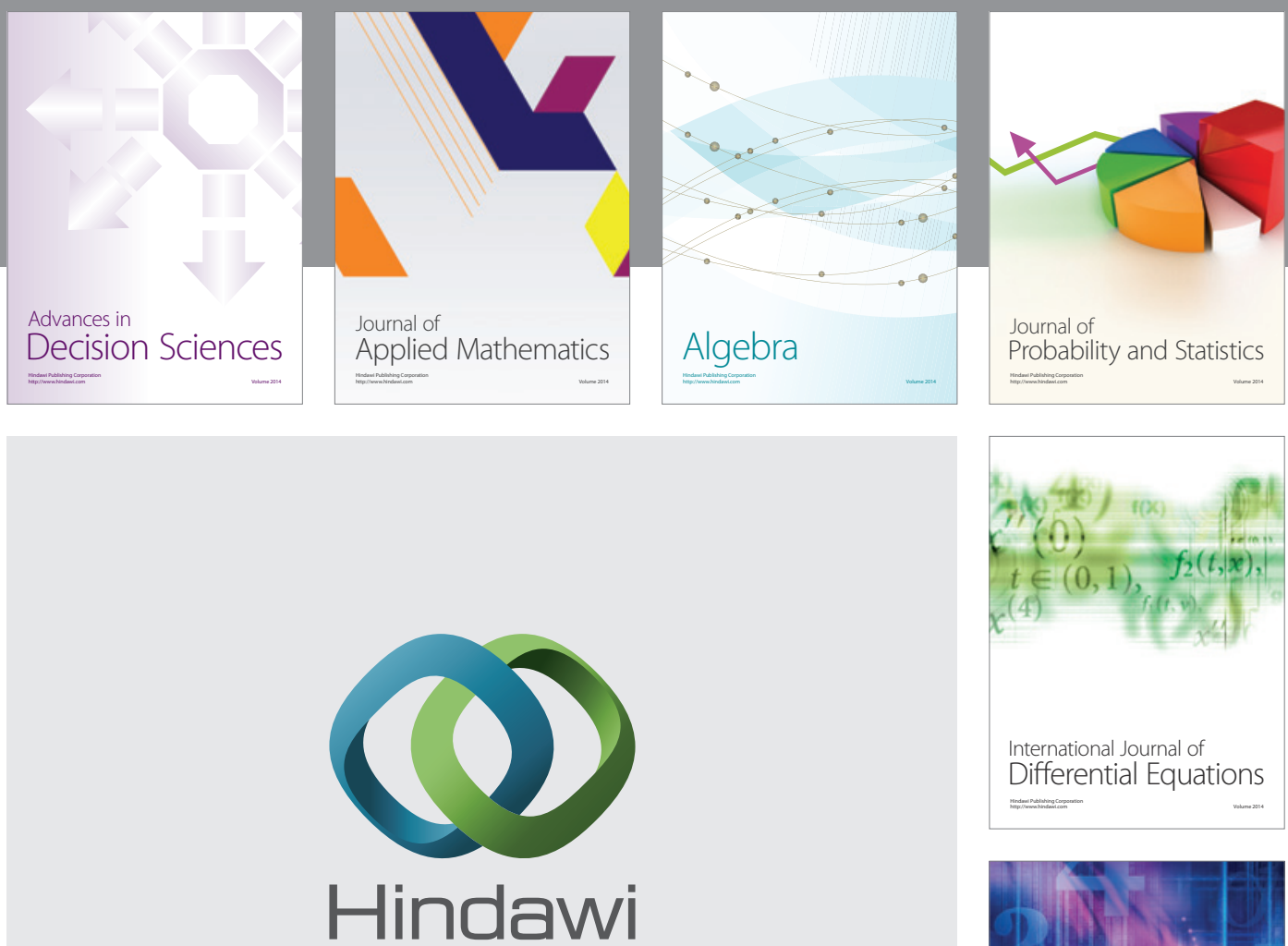

Submit your manuscripts at http://www.hindawi.com
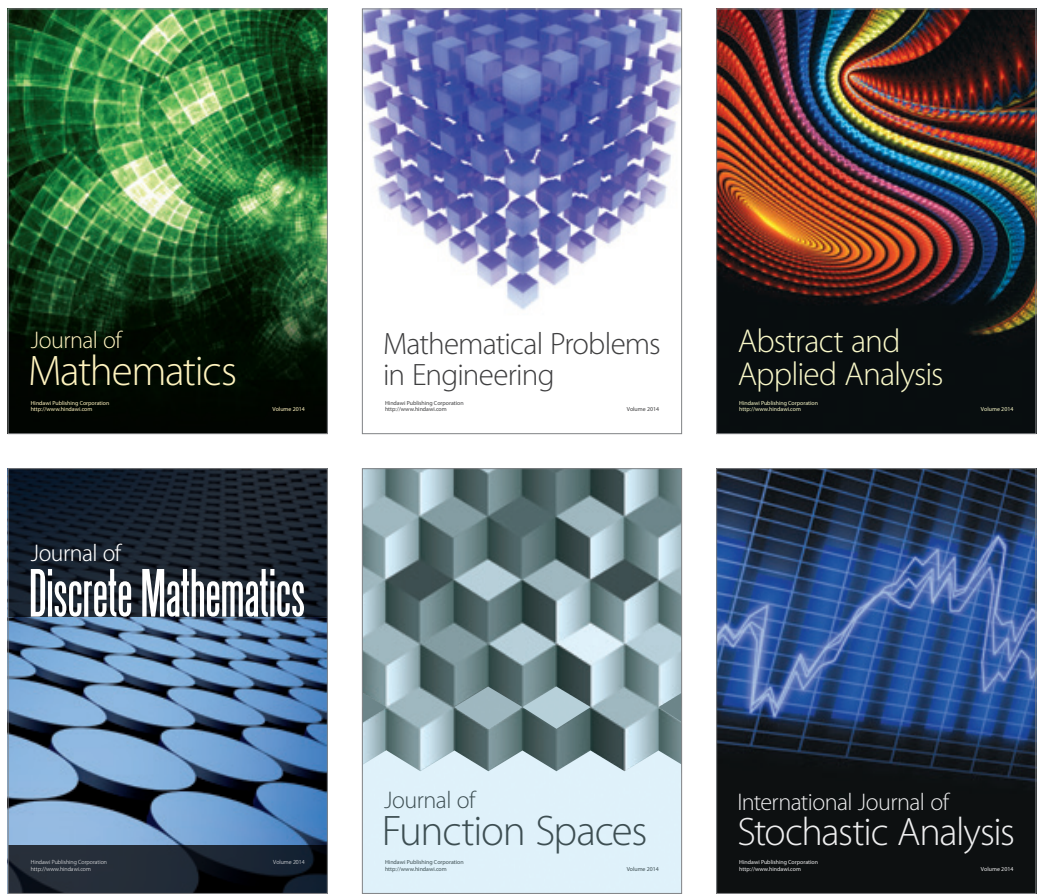

Journal of

Function Spaces

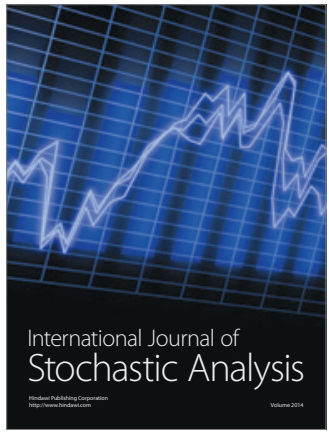

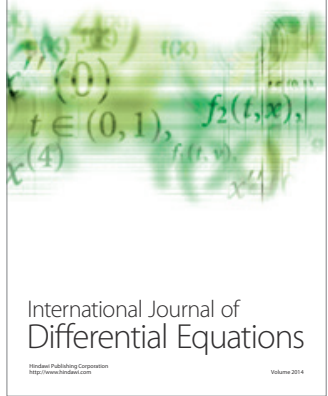
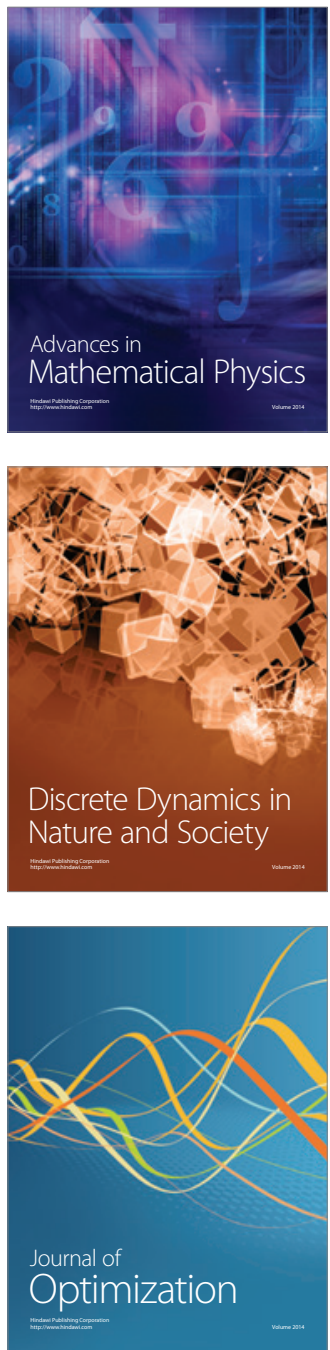